\title{
Trichomonas Vaginalis Inhibits HeLa Cell Growth Through Modulation of Critical Molecules for Cell Proliferation and Apoptosis
}

\author{
ZIWEN ZHU ${ }^{1,2^{*}}$, LEI ZHAO ${ }^{3 *}$, ANDREW BRITTINGHAM ${ }^{1}$, \\ QIAN BAI $^{2}$, MARK R. WAKEFIELD ${ }^{2}$ and YUJIANG FANG ${ }^{1,2}$ \\ ${ }^{1}$ Department of Microbiology, Immunology \& Pathology, \\ Des Moines University College of Osteopathic Medicine, Des Moines, IA, U.S.A.; \\ ${ }^{2}$ Department of Surgery, University of Missouri School of Medicine, Columbia, MO, U.S.A.; \\ ${ }^{3}$ Department of Respiratory Medicine, the Second People's Hospital of Hefei and Hefei \\ Hospital Affiliated to Anhui Medical University, Hefei, P.R. China
}

\begin{abstract}
Background/Aim: Cervical cancer is one of the deadliest gynecological cancers in USA. The role of Trichomonas Vaginalis (T. Vag) in the etiology or pathogenesis of cervical cancer is still poorly understood and controversial. Materials and Methods: Clonogenic assay, PCNA staining, TUNEL staining and caspase-3 activity assay were used to investigate the direct in vitro effect of T. Vag on human cervical cancer by using HeLa cells. We further investigated the potential molecular mechanisms using RT-PCR and immunohistochemical staining. Results: We found that culture supernatant of T. Vag inhibited growth of HeLa cervical cancer cells and this correlated with up-regulation of p15. We also found that culture supernatant of T. Vag induced apoptosis of HeLa cells and this correlated with up-regulation of Fas, TRAIL and TRAILR1. Conclusion: Culture supernatant of T. Vag inhibits growth of HeLa cervical cancer cells by inhibition of proliferation and promotion of apoptosis. Our study might be helpful to address the association between the development of cervical cancer and infection of $T$. Vag.
\end{abstract}

Cervical cancer is a malignancy arising from the cervix that could invade surrounding tissues and metastasize to other

*These Authors contributed equally to this study.

Correspondence to: Dr. Yujiang Fang, Department of Microbiology, Immunology \& Pathology, Des Moines University College of Osteopathic Medicine, Des Moines, IA 50312, U.S.A. Tel: +1 5152711435, Fax: +1 5152711543, e-mail: yujiang.fang@dmu.edu

Key Words: Trichomonas Vaginalis, cervical cancer, apoptosis, proliferation, p15, TRAILR1. organs. It is one of the deadliest gynecological cancers in the United States and worldwide. In the United States in 2016, there are about 13,000 estimated new cases and over 4,000 estimated deaths due to cervical cancer (1). Worldwide, there are over 500,000 new cases and 274,000 deaths reported each year (1). The prognosis for cervical cancer is very challenging. The five year survival rate for women with cervical cancer is less than $50 \%$ in developing countries, and about $66 \%$ in developed countries (2). To improve the prognosis of cervical cancer, it is critical to understand the etiology and the pathogenesis of the disease. Unfortunately, little progress has been made in recent years regarding the etiology or pathogenesis of cervical cancer. Trichomonas vaginalis (T. Vag) is an anaerobic, flagellated extracellular protozoan parasite (3). It is well-known that it is the most common non-viral sexually transmitted pathogen worldwide, with an incidence of 275 million cases annually (4). In the Unites States alone, 8-10 million estimated new infections occur annually (5). Despite the fact that only $50 \%$ of infected women will show clinical symptoms, the clinical significance of this infection lies in the fact that it increases susceptibility to HIV, infertility, premature labor, and possibly a higher incidence of cervical cancer (6).

T. Vag has been suspected to be associated with the pathogenesis of cervical cancer for more than twenty years (7-10). However, increasing evidence seems to suggest that T. Vag might not be associated with the development of cervical cancer (11-13). Consistent with these findings, our previous study has also shown that culture supernatant of $T$. Vag inhibits growth of prostate cancer cells through upregulation of the anti-proliferative molecule p21 and downregulation of the anti-apoptotic molecule Bcl-2 (14). Interestingly, one study even suggests that $T$. Vag can damage normal genital epithelial cells and induce apoptosis 
of Siha cervical cancer cells through the Bcl-2 family (15). Obviously, the association between $T$. Vag and cervical cancer is not fully understood and further studies are needed to clarify their relationship. This study was designed and performed to investigate the direct effect of $T$. Vag on HeLa cell growth by using the culture supernatant of $T$. Vag.

\section{Materials and Methods}

Cervical cancer cell culture. Human cervical cancer cell line, HeLa, was obtained from the American Type Culture Collection (Manassas, VA, USA). HeLa cervical cancer cells were cultured in DMEM medium in the presence of $10 \%$ FBS as well as $1 \%$ penicillin-streptomycin (Invitrogen, Carlsbad, CA, USA). HeLa cervical cancer cells were cultured at $37^{\circ} \mathrm{C}$ in $5 \% \mathrm{CO}_{2}$ humidified incubator. HeLa cancer cells were used for the designed experiment when they reached $70 \%$ confluence.

T. Vag cell culture. Trichomonas vaginalis G3 (ATCC PRA-98) was obtained from the American Type Culture Collection. The culture method has been described in detail in our previous study (14).

Collection of T. Vag conditioned growth medium. Following $48 \mathrm{~h}$ of growth, cultures reached an average density of $3.5 \times 10^{6}$ cells $/ \mathrm{ml}$. Conditioned media of cultured $T$. Vag were obtained by centrifugation. Post-centrifugation, conditioned medium without any cell debris was immediately frozen using liquid nitrogen and stored at $-80^{\circ} \mathrm{C}$. Control media, which had not been inoculated with $T$. Vag, was processed in an identical manner.

Treatment of cervical cancer cells with T. Vag conditioned growth medium. $70 \%$ confluent HeLa cells were treated for 3 days with conditioned growth medium or control medium alone. The dilution ratio for $T$. Vag conditioned growth medium used in this study is based on our previous study (14). A dilution of 1:15 for T. Vag conditioned growth medium was used in this study.

Immunohistochemistry (IHC). IHC staining for PCNA, p15 and TRAILR1 was performed as previously described (16-20). Average staining intensity for proteins within the area covered by cells was measured using ImageJ software. Results are expressed as the average integrated immunostaining intensity of 3 slides \pm SEM relative to that in control cells.

Clonogenic survival assay. Three days after T. Vag. conditioned growth medium treatment, cancer cells were detached and counted in a hemocytometer. Clonogenic survival assay was performed as described previously (16-20). The number of colonies was counted and expressed as a percentage of total colonies in control.

RT-PCR. HeLa cancer cells were washed with PBS and homogenized in TRIzol (Invitrogen). RNA was extracted and its concentration was determined by Nanodrop, then $1 \mu \mathrm{g}$ RNA was reverse transcribed. GAPDH was used as a housekeeping gene. Primer sequences used have been described previously (16).

TUNEL staining. Apoptosis was determined by TUNEL assay using an ApopTag kit (Chemicon, El Segundo, CA, USA) according to the manufacturer's instructions which has been described in detail in our previous studies (16-20). To quantify the number of apoptotic cells, all cells in 5-6 randomly selected high-power fields (magnification: $\times 400$ ) were manually counted. TUNEL+ cells were expressed as a percentage of total cells.

Measurement of caspase-3 activity. As we have described in our previous studies (16-20), cellular caspase-3 activity of HeLa cells was measured using a Caspase-3/CPP32 Colorimetric Assay kit (BioVision, Milpitas, CA, USA) according to the manufacturer's instructions.

Statistics. All experiments were repeated at least two times. Comparisons between 2 groups were performed using a 2-tailed unpaired Student's $t$-test. A $p$-value $<0.05$ was considered significant.

\section{Results}

Proliferation of HeLa cervical cancer cells was inhibited by culture supernatant of T. Vag. HeLa cell line is the most commonly used cell line in cervical cancer research. In this study, to investigate the direct effect of $T$. Vag supernatant on proliferation of cervical cancer. 70\% confluent HeLa cells were treated with culture supernatant of $T$. Vag or control medium alone for 3 days and cell survival was evaluated by the traditional clonogenic survival assay. As shown in Figure 1A, the percentage of colonies of HeLa cells after $T$. Vag treatment was significantly lower than that in control treated cells with medium alone (Figure 1A, $p<0.05$ ). To further confirm this finding, IHC for PCNA, which is a marker of cell proliferation, was also used to evaluate the direct effect of the culture supernatant of T. Vag on HeLa cells. Consistent with the finding revealed by the clonogenic survival assay, immunohistostaining intensity for PCNA in cells after T. Vag treatment was significantly weaker than that in control (Figure 1B and C). Taken together, these results indicate that proliferation of HeLa cells was inhibited by the culture supernatant of $T$. Vag.

Culture supernatant of T. Vag up-regulated expression of anti-proliferative molecule p15 in HeLa cells. Cell cycle is mainly determined by the balance between pro- and antiproliferative molecules. When pro-proliferative molecules dominate, cells start to proliferate. Whereas, when antiproliferative molecules dominate, cell cycle arrest is observed. To investigate the possible molecular mechanisms by which proliferation of HeLa cells was inhibited by culture supernatant of T. Vag, mRNA expression of important proand anti-proliferative molecules was first determined by RTPCR in both groups (Figure 2). The mRNA expression of these molecules was all comparable in both groups $(p>0.05)$ except that of p15 whereby its mRNA expression level was significantly higher in $T$. Vag group than that in the control group (Figure 2, $p<0.05$ ). This finding was further supported by IHC staining for anti-proliferative protein $\mathrm{p} 15$. The staining intensity for p15 was much stronger in $T$. Vag group than in the control group (Figure 3). Thus, these data 

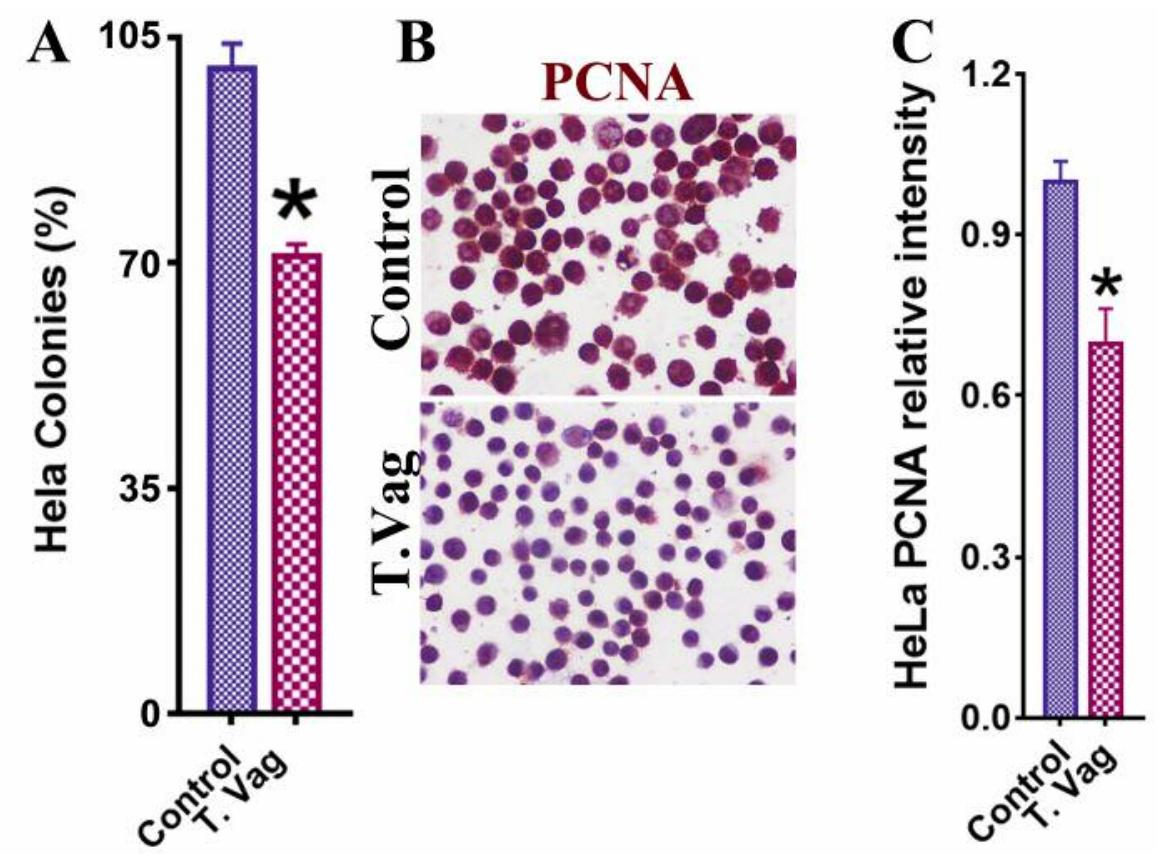

Figure 1. Proliferation of HeLa cervical cancer cells was inhibited by culture supernatant of T. Vag. A: The clonogenic survival data for HeLa cells treated with T. Vag conditioned growth medium are shown. The number of colonies was counted and expressed as a percentage of total colonies in control (medium alone). B-C: Shown are IHC results for PCNA and staining intensity of HeLa cells treated with culture supernatant of T. Vag or medium alone. A significant difference in the percentage of colonies or staining intensity in each group compared to that in control is indicated by the asterisk $(p<0.05)$. Original magnification in $B: \times 400$.

strongly indicated that up-regulation of p15 correlated with the inhibitory effect of $T$. Vag on proliferation of HeLa cells

Culture supernatant of T. Vag induced apoptosis of HeLa cells. In our previous study (14), we found that the growth inhibitory effect of $T$. Vag on prostate cancer cells was also attributed to the apoptosis inducing effect by $T$. Vag. To address this possibility, $70 \%$ confluent HeLa cells were treated with culture supernatant of $T$. Vag or control medium alone for 3 days and apoptosis was evaluated by using two different methods, TUNEL staining (Figure 4A) and caspase-3 activity kit (Figure 4C). We found that the number of TUNEL ${ }^{+}$cells in the group treated with culture supernatant of $T$. Vag was much higher than that in the control group and this difference was significant (Figure 4B, $p<0.05$ ). To confirm this finding, apoptosis was evaluated by using caspase- 3 activity kit, which showed that the relative caspase- 3 activity in the group treated with culture supernatant of $T$. Vag was also significantly higher than that in the control group (Figure $4 \mathrm{C}, p<0.05$ ). Clearly, these data indicated that T. Vag induced apoptosis of HeLa cells.

Culture supernatant of T. Vag up-regulated the expression of pro-apoptotic molecules in HeLa cells. We have previously shown that apoptosis is the result of an imbalance of pro- and anti-apoptotic molecules which favors the direction of apoptosis (14). To further investigate the potential molecular mechanisms by which T. Vag induced apoptosis of HeLa cells, mRNA expression of important pro- and anti-apoptotic molecules in HeLa cells was determined by RT-PCR. As shown in Figure 5, the mRNA expression of these molecules was comparable in both groups $(p>0.05)$ except for Fas, TRAIL, TRAILR1 and FLIP. Their mRNA expression level was significantly higher in $T$. Vag group than that in the control group (Figure 5, $<<0.05$ ). In fact, the seemingly contradictory up-regulation of the anti-apoptotic molecule FLIP was not a surprise since a similar result had been observed in our previous studies $(16,21-23)$. Due to limited slides available, we randomly chose to perform IHC for TRAILR1 to confirm the data revealed by RT-PCR at a protein level. Results from IHC staining for TRAILR1 further supported the finding by RT-PCR (Figure 6). These results indicated up-regulation of pro-apoptotic molecule TRAILR1 as well as Fas and TRAIL correlated with the increased apoptosis in HeLa cells induced by T. Vag.

\section{Discussion}

This study focused on whether T. Vag. has any direct effect on proliferation and/or apoptosis of the HeLa cell line. We 

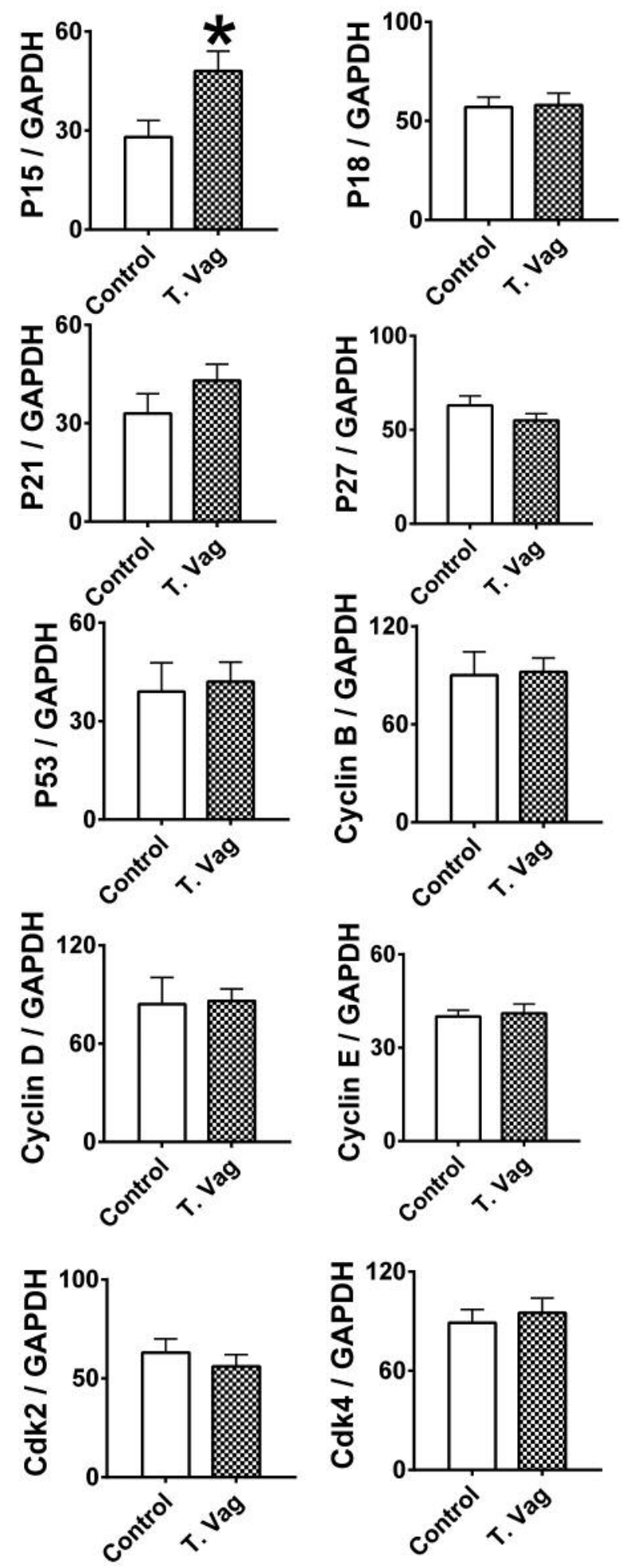

Figure 2. Effect of culture supernatant of T. Vag on expression of proand anti-proliferative molecules evaluated by RT-PCR. Results are expressed as the mean ratio of pro- and anti-proliferative molecule densitometric Units/GAPDH + SEM $(\times 100)$. A significant difference in mRNA expression between HeLa cells treated with culture supernatant of T. Vag and those in control is indicated by the asterisk $(p<0.05)$.

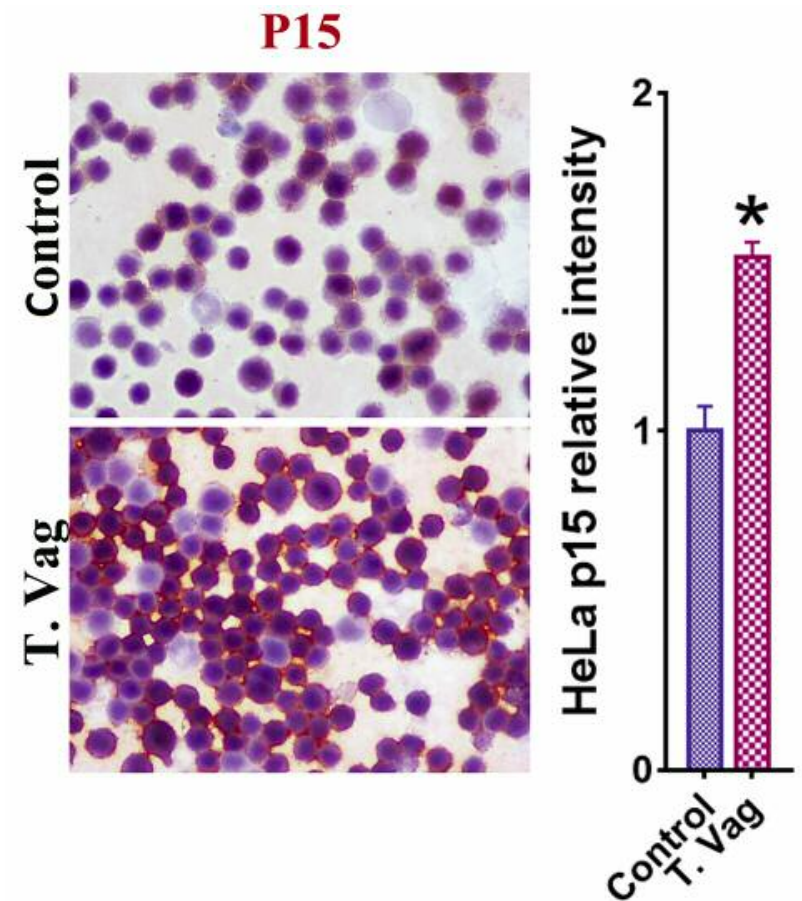

Figure 3. Effect of culture supernatant of T. Vag on the expression of p15 evaluated by IHC. Representative pictures of IHC and the relative staining intensity are shown. Results are expressed as the average integrated staining intensity relative to that in control. A significant difference in staining intensity between cells treated with culture supernatant of T. Vag and those in control is indicated by the asterisk $(p<0.05)$. Original magnification: $\times 400$.

found that culture supernatant of $T$. Vag inhibited the growth of cervical cancer cells and its potential mechanism might be correlated with up-regulation of p15, TRAILR1 as well as Fas and TRAIL. This study extends our previous study showing that $T$. Vag inhibited growth of prostate cancer cells and suggests that the growth inhibitory effect of $T$. Vag on cancer cells is not only limited to a type of cancer. To the best of our knowledge, our study is the first to directly demonstrate that there is a negative association between growth of HeLa cells and infection of T. Vag.

It has been more than 20 years since the first study proposed that there might be an association between $T$. Vag and cervical cancer (7). In the years that followed, some other studies supported this proposition. These studies suggested that $T$. Vag was related to a higher risk for the development of cervical cancer of a higher pathological grade and a more invasive type $(7,8,11)$. The presence of $T$. Vag was even proposed to be a predictor for cervical cancer $(7,8,11)$. These studies have been challenged by a study published in 2013 which proposed that $T$. Vag was not associated with promotion of HSIL and cervical cancer at all (13). Interestingly, the data 

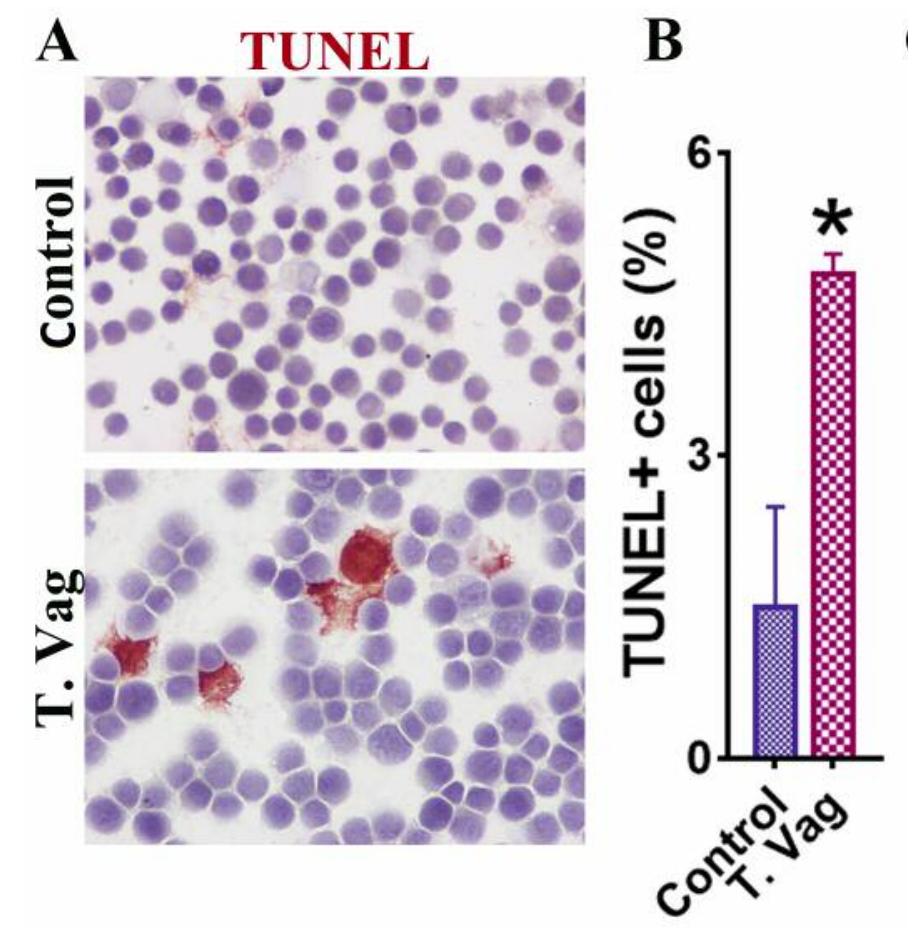

C

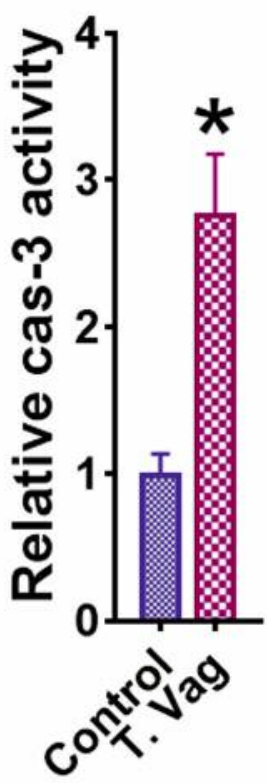

Figure 4. Culture supernatant of T. Vag induced apoptosis of HeLa cells. A and B: Representative TUNEL staining and TUNEL+ cell counting are shown. C: Shown is cellular caspase-3 activity. Results are expressed as mean activity relative to control + SEM. A significant difference in the percentage of TUNEL+ cells or relative caspase-3 activity in cells treated with culture supernatant of T. Vag and those in control is indicated by the asterisk $(p<0.05)$. Original magnification: A: $\times 400$.

presented here also challenge the concept that there might be a positive association between $T$. Vag and cervical cancer. In this study, we showed that culture supernatant of $T$. Vag inhibited the growth of HeLa cells in vitro, suggesting that $T$. Vag might inhibit growth of cervical cancer. In fact, our study is consistent with a study showing that $T$. Vag promoted apoptosis of cervical cancer cells by using Siha cells (15).

Cell proliferation is a process resulting in an increased number of cells. Cell proliferation is elaborately regulated by the balance between pro- and anti-apoptotic molecules (24-27). One of the hallmarks of neoplasia is uncontrolled proliferation (28). Cyclin B, D, E and cyclin-dependent kinase 2 and 4 (cdk2 and cdk4) are regarded as proproliferative molecules because they promote cell proliferation (29). Whereas, p15, p18, p21, p27 and p53 are traditionally regarded as anti-proliferative molecules because they inhibit cell proliferation $(24,25,30)$. In this study, we found that the anti-proliferative effect of the cultured supernatant of $T$. Vag correlated with increased expression of p15. It is well-known that p15 is a critical anti-proliferative molecule encoded by CDKN2B that is frequently mutated and deleted in a variety of tumors such as acute lymphoblastic leukemia and melanoma (31-33). p15 is a cyclin-dependent kinase inhibitor, also known as p15Ink4b, which can form a complex with cdk4 or cdk6, and prevents the activation of the CDK kinases by cyclin D to inhibit cells from progressing from $\mathrm{G} 1$ to $\mathrm{S}$ phase $(31,34)$. As a result, up-regulation of this molecule would arrest cell cycle and subsequently result in cell growth inhibition. As shown in our study, T. Vag significantly increased p15 which was evidenced at both mRNA and protein levels. Thus, it is reasonable that the growth and proliferation of HeLa cervical cancer cells are inhibited in the presence of culture supernatant of T. Vag. In this study, among the the pro- and anti- proliferative molecules examined, only the expression level of p15was altered by following treatment with the culture supernatant of $T$. Vag, suggesting that p15 is a key target of T. Vag in HeLa cells.

The number of cell in tissues is not only determined by cell proliferation, but also by cell apoptosis (28). Apoptosis is mediated by the sequential activation of caspases and caspase- 3 is a critical caspase activated in apoptosis $(35,36)$. Similar to TUNEL staining which detects the break of DNA, caspase-3 activity has also been regarded as a marker for apoptosis. In this study, besides TUNEL staining, an increased activity for caspase-3 was detected in HeLa cells after treatment with the culture supernatant of T. Vag. This strongly indicates the contribution of apoptosis to constrain the growth of HeLa 

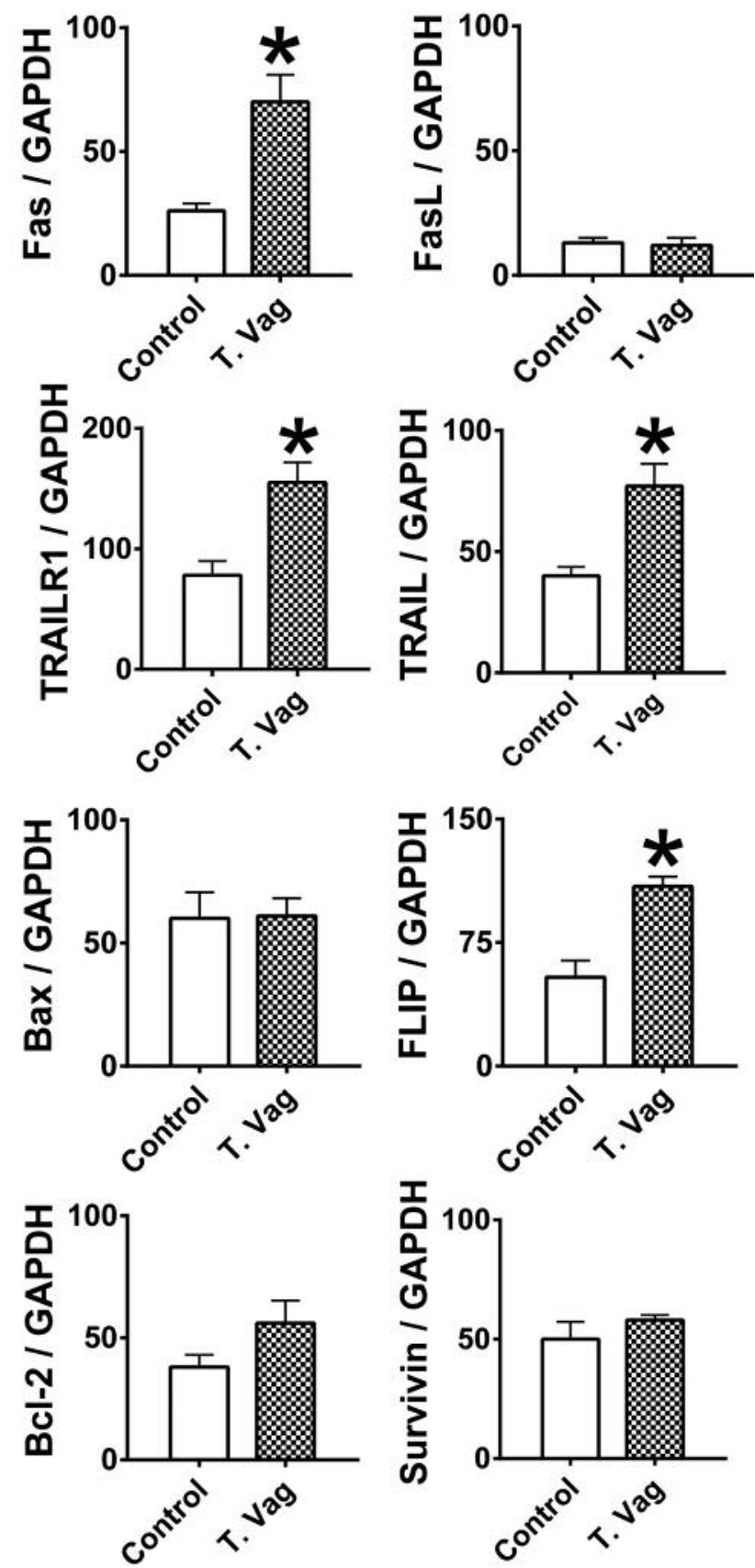

Figure 5. Effect of culture supernatant of T. Vag on expression of proand anti-apoptotic molecules evaluated by RT-PCR Results are expressed as the mean ratio of pro- and anti-apoptotic molecule densitometric Units/GAPDH + SEM (x100). A significant difference in mRNA expression between cells treated with culture supernatant of T. Vag and those in control is indicated by the asterisk $(p<0.05)$.

cancer cells. Apoptosis is also a fine-tuned process orchestrated by the balance between pro- and anti-apoptotic molecules. Traditionally, Fas, FasL, TRAILR1, TRAIL and Bax are categorized as pro-apoptotic molecules because they

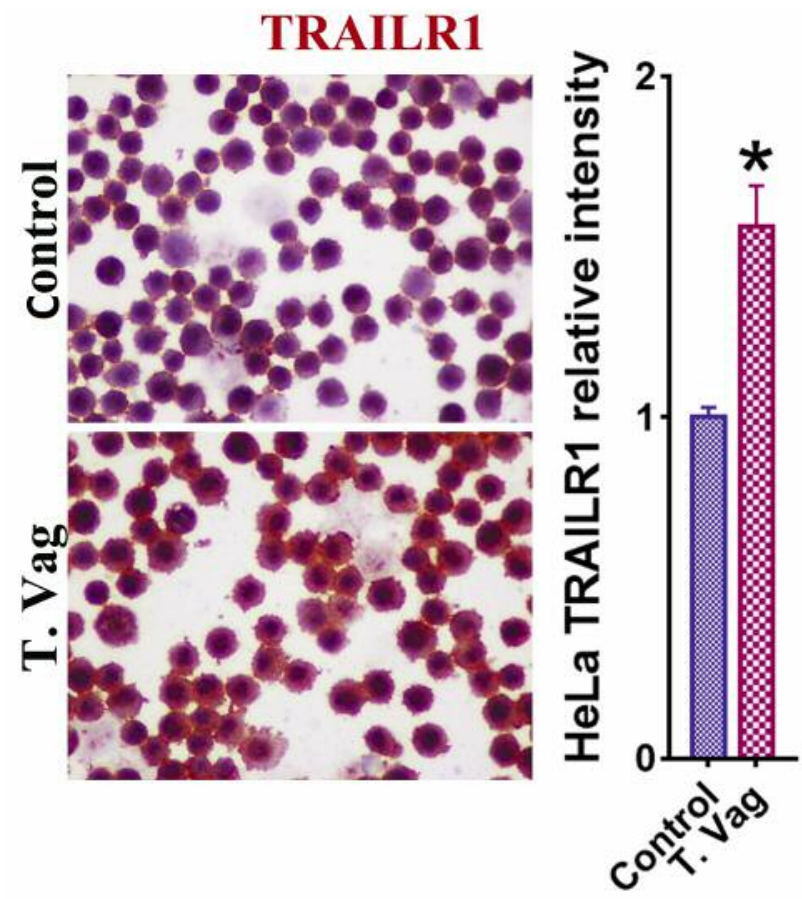

Figure 6. Effect of culture supernatant of T. Vag on expression of TRAILRI evaluated by IHC. Representative pictures of IHC and relative staining intensity are shown. Results are expressed as the average integrated staining intensity relative to that in control cells. A significant difference in staining intensity between cells treated with culture supernatant of $T$. Vag and those in control is indicated by the asterisk $(p<0.05)$. Original magnification: $\times 400$.

promote cell apoptosis, whereas, FLIP, Bcl-2 and survivin are traditionally categorized as anti-apoptotic molecules because they inhibit apoptosis (18). Apoptosis could be triggered by two pathways, the extrinsic and intrinsic pathway. Both Fas/FasL and TRAIL/TRAILR signals induce apoptosis through classic extrinsic apoptotic pathway, that eventually leads to the activation of sequential caspases and induction of apoptosis (37). In this study, we showed a significant increase in the expression level of pro-apoptotic molecules TRAILR1, TRAIL as well as Fas in the T. Vag group. This suggests that T. Vag induces apoptosis of HeLa cervical cancer cells, not just by targeting one pro-apoptotic molecule, but by targeting multiple pro-apoptotic molecules. It has been shown that TRAIL/TRAILR pathway is one of the critical pathways for cancer cells to undergo apoptosis (37). TRAIL/TRAILR signal pathway is also targeted by cytokines when they induce apoptosis. We have reported that IL-9 and IL-32 promoted apoptosis by up-regulation of TRAILR 1 (21, 22). In our previous study (14), culture supernatant of $T$. Vag promoted apoptosis through down-regulation of anti-apoptotic molecule Bcl-2, but not through modulation of other pro- or antiapoptotic molecules. Furthermore, T. Vag. has been shown to induce apoptosis in $\mathrm{SiHa}$ cells through intrinsic pathway by 
the mechanisms of the dissociation of Bcl-xL/Bim and Mcl1/Bim complexes (15). All of these strongly suggest that the molecular mechanism by which T. Vag promotes apoptosis is complicated and might be cell line specific.

In this study, the expression of FLIP was found to be upregulated by the culture supernatant of $T$. Vag. In fact, this is not a surprise to us at all since this seemingly contradictory finding has also been noticed in our previous studies $(16,21$ $23)$. The detailed possible mechanisms for this are still under investigation. We attribute this to an adaptive response of cancer cells to cell injury so that further damage to cancer cells could be minimized. Nevertheless, despite the fact that higher expression of FLIP induced by T. Vag in HeLa cancer cells may favor the survival of cancer cells, the overwhelming higher expression of Fas, TRAIL as well as TRAILR 1 is predominating. This eventually results in the direction of apoptosis for HeLa cancer cells after treatment with supernatant of $T$. Vag.

\section{Conclusion}

In summary, T. Vag inhibits the growth of HeLa cells by modulation of p15, TRAILR1, TRAIL and Fas, all of which are critical molecules in cell proliferation or apoptosis.

\section{Conflicts of Interest}

The Authors have no conflicts of interest regarding this study.

\section{Acknowledgements}

This study was supported by grants for Yujiang Fang (Iowa Science Foundation Grant ISF 16-8, IOER 05-14-01, IOER 112-3749 and IOER 112-3104).

\section{References}

1 Siegel RL, Miller KD and Jemal A: Cancer statistics, 2016. CA Cancer J Clin 66(1): 7-30, 2016.

2 Franco EL, Schlecht NF and Saslow D: The epidemiology of cervical cancer. Cancer J 9(5): 348-359, 2003.

3 Dirkx M, Boyer MP, Pradhan P, Brittingham A and Wilson WA: Expression and characterization of a $\beta$-fructofuranosidase from the parasitic protist trichomonas vaginalis. BMC Biochem 15(1): 1, 2014.

4 Schwebke JR and Burgess D: Trichomoniasis. Clin Microbiol Rev 17(4): 794-803, 2004.

5 McClelland RS: Trichomonas vaginalis infection: Can we afford to do nothing? J Infect Dis 197(4): 487-489, 2008.

6 Hirt RP and Sherrard J: Trichomonas vaginalis origins, molecular pathobiology and clinical considerations. Curr Opin Infect Dis 28(1): 72-79, 2015.

7 Gram IT, Macaluso M, Churchill J and Stalsberg H: Trichomonas vaginalis (TV) and human papillomavirus (HPV) infection and the incidence of cervical intraepithelial neoplasia (CIN) grade III. Cancer Causes Control 3(3): 231-236, 1992.
8 Yap E, Ho T, Chan Y, Thong T, Ng G, Ho L and Singh M: Serum antibodies to trichomonas vaginalis in invasive cervical cancer patients. Genitourin Med 71(6): 402-404, 1995.

9 Zhang Z-F, Graham S, Yu S-Z, Marshall J, Zielezny M, Chen Y$\mathrm{X}$, Sun M, Tang S-L, Liao C-S and Xu J-L: Trichomonas vaginalis and cervical cancer: A prospective study in china. Ann Epidemiol 5(4): 325-332, 1995.

10 Sayed e-AS, El-Wakil H, Kamel N and Mahmoud M: A preliminary study on the relationship between trichomonas vaginalis and cervical cancer in egyptian women. J Egypt Soc Parasitol 32(1): 167-178, 2002.

11 Viikki M: Gynaecological infections as risk determinants of subsequent cervical neoplasia. Acta Oncol 39(1): 71-75, 2000.

12 Zhang Z-F and Begg CB: Is trichomonas vaginalis a cause of cervical neoplasia? Results from a combined analysis of 24 studies. Int J Epidemiol 23(4): 682-690, 1994.

13 Donders GG, Depuydt CE, Bogers J-P and Vereecken AJ: Association of trichomonas vaginalis and cytological abnormalities of the cervix in low risk women. PloS One 8(12): e86266, 2013.

14 Zhu Z, Davidson KT, Brittingham A, Wakefield MR, Bai Q, Xiao $\mathrm{H}$ and Fang Y: Trichomonas vaginalis: A possible foe to prostate cancer. Med Oncol 33(10): 115, 2016.

15 Quan J-H, Kang B-H, Cha G-H, Zhou W, Koh Y-B, Yang J-B, Yoo H-J, Lee M-A, Ryu J-S and Noh H-T: Trichonomas vaginalis metalloproteinase induces apoptosis of siha cells through disrupting the mcl-1/bim and bcl-xl/bim complexes. PloS One 9(10): e110659, 2014.

16 Fang Y, DeMarco VG and Nicholl MB: Resveratrol enhances radiation sensitivity in prostate cancer by inhibiting cell proliferation and promoting cell senescence and apoptosis. Cancer Sci 103(6): 1090-1098, 2012.

17 Fang Y, Wei Y, DeMarco V, Chen K, Sharp GC and BraleyMullen H: Murine flip transgene expressed on thyroid epithelial cells promotes resolution of granulomatous experimental autoimmune thyroiditis in dba/1 mice. Am J Pathol 170(3): 875887, 2007.

18 Fang Y and Braley-Mullen H: Cultured murine thyroid epithelial cells expressing transgenic fas-associated death domain-like interleukin-1 $\beta$ converting enzyme inhibitory protein are protected from fas-mediated apoptosis. Endocrinology 149(7): 3321-3329, 2008.

19 Fang Y, Sharp GC, Yagita H and Braley-Mullen H: A critical role for trail in resolution of granulomatous experimental autoimmune thyroiditis. J Pathol 216(4): 505-513, 2008.

20 Fang Y, Yu S and Braley-Mullen H: Tgf- $\beta$ promotes proliferation of thyroid epithelial cells in ifn- $\gamma^{-/-}$mice by down-regulation of p21 and p27 via akt pathway. Am J Pathol 180(2): 650-660, 2012.

21 Nicholl MB, Chen X, Qin C, Bai Q, Zhu Z, Davis MR and Fang Y: Il-32 $\alpha$ has differential effects on proliferation and apoptosis of human melanoma cell lines. J Surg Oncol 113(4): 364-369, 2016.

22 Fang Y, Chen X, Bai Q, Qin C, Mohamud AO, Zhu Z, Ball TW, Ruth CM, Newcomer DR and Herrick EJ: Il-9 inhibits htb-72 melanoma cell growth through upregulation of p21 and trail. J Surg Oncol 111(8): 969-974, 2015.

23 Nicholl MB, Ledgewood CL, Chen X, Bai Q, Qin C, Cook KM, Herrick EJ, Diaz-Arias A, Moore BJ and Fang Y: Il-35 promotes pancreas cancer growth through enhancement of proliferation and inhibition of apoptosis: Evidence for a role as an autocrine growth factor. Cytokine 70(2): 126-133, 2014. 
24 Johnson D and Walker C: Cyclins and cell cycle checkpoints. Annu Rev Pharmacol Toxicol 39(1): 295-312, 1999.

25 Ohtsubo M, Theodoras AM, Schumacher J, Roberts JM and Pagano M: Human cyclin e, a nuclear protein essential for the g1-to-s phase transition. Mol Cell Biol 15(5): 2612-2624, 1995.

26 Sherr CJ: G1 phase progression: Cycling on cue. Cell 79(4): 551-555, 1994.

27 Sherr CJ: D-type cyclins. Trends Biochem Sci 20(5): 187-190, 1995.

28 Hanahan D and Weinberg RA: Hallmarks of cancer: The next generation. Cell 144(5): 646-674, 2011.

29 Zhang D, Li X, Chen C, Li Y, Zhao L, Jing Y, Liu W, Wang X, Zhang Y, Xia H, Chang Y, Gao X, Yan J and Ying $\mathrm{H}$ : Attenuation of $\mathrm{p} 38$-mediated mir-1/133 expression facilitates myoblast proliferation during the early stage of muscle regeneration. PLoS One 7(7): e41478, 2012.

30 Zhu Z, Zhang D, Lee H, Menon AA, Wu J, Hu K and Jin Y: Macrophage-derived apoptotic bodies promote the proliferation of the recipient cells via shuttling microrna-221/222. J Leukoc Biol 101(6): 1349-1359, 2017.

31 Krimpenfort P, IJpenberg A, Song J-Y, van der Valk M, Nawijn $\mathrm{M}$, Zevenhoven $\mathrm{J}$ and Berns A: P15ink4b is a critical tumour suppressor in the absence of p16ink4a. Nature 448(7156): 943946, 2007.
32 De Braekeleer M, Douet-Guilbert N and De Braekeleer E: Prognostic impact of p15 gene aberrations in acute leukemia. Leuk Lymphoma 58(2): 257-265, 2017.

33 McNeal AS, Liu K, Nakhate V, Natale CA, Duperret EK, Capell BC, Dentchev T, Berger SL, Herlyn M and Seykora JT: Cdkn2b loss promotes progression from benign melanocytic nevus to melanoma. Cancer Discov 5(10): 1072-1085, 2015.

34 Hannon GJ and Beach D: P15ink4b is a potential effector of tgfbeta-induced cell cycle arrest. Nature 371(6494): 257-261, 1994.

35 Griffith TS, Brunner T, Fletcher SM, Green DR and Ferguson TA: Fas ligand-induced apoptosis as a mechanism of immune privilege. Science 270(5239): 1189-1192, 1995.

36 Thompson CB: Apoptosis in the pathogenesis and treatment of disease. Science 267(5203): 1456, 1995.

37 Johnstone RW, Frew AJ and Smyth MJ: The trail apoptotic pathway in cancer onset, progression and therapy. Nat Rev Cancer 8(10): 782-798, 2008.
Received July 5, 2018

Revised July 23, 2018

Accepted July 24, 2018 